\title{
The influence of cardiac resynchronization therapy on subjective and objective parameters of sleep, and their association with the function of the autonomous nervous system
}

\author{
Anna Przybyła, Danuta Czarnecka \\ $1^{\text {st }}$ Department of Cardiology, Interventional Electrocardiology and Arterial Hypertension, Jagiellonian University Medical College, Krakow, \\ Poland
}

Adv Interv Cardiol 2019; 15, 3 (57): 357-363

DOI: https://doi.org/10.5114/aic.2019.87892

\begin{abstract}
A bstract
Introduction: Cardiac resynchronization therapy (CRT) was a breakthrough in the treatment of heart failure, but data regarding the effect of this therapy on numerous disorders associated with heart failure are limited.

Aim: To assess the impact of CRT on sleep breathing disorders, and to determine the relationship between the changes in the autonomous nervous system and sleep disorders after CRT.

Material and methods: The study included 55 patients with chronic heart failure stable for at least last 3 months, in New York Heart Association (NYHA) class III or IV despite optimal medical therapy, with a reduced left ventricular ejection fraction (LVEF) $\leq 35 \%$, QRS complex duration $\geq 120 \mathrm{~ms}$, and sinus rhythm. Before and 3 months after implementation of CRT echocardiography, 6-minute walk test (6MWT), polysomnography with the Pittsburgh Sleep Quality Index (PSQI) questionnaire and the Epworth Sleepiness Scale (ESS) were performed. Also baroreflex sensitivity (BRS) was evaluated.

Results: After implementation of CRT, the values of the apnea-hypopnea index (AHI), apnea index (AI), and central and mixed apnea indexes (CAI, MAI) were statistically significantly reduced. The strongest negative correlations were demonstrated for changes in CAI and changes in BRS. An improvement in sleep quality, daytime sleepiness, LVEF, NYHA class, and 6MWT was observed and was the most strongly associated with the improvement in CAI, too.

Conclusions: CRT has a beneficial effect on subjective and objective features of sleep, as well as on the function of the autonomous nervous system. In addition, patients with heart failure and coexisting central sleep apnea may benefit most from this therapy.
\end{abstract}

Key words: heart failure, sleep-disordered breathing, biventricular pacing, baroreflex.

S u m m a ry

The publication concerns the assessment of sleep breathing disorders as well as their interactions with the function of the autonomous nervous system after cardiac resynchronization therapy (CRT). After a 3-month observation period, the improvement in sleep breathing disorders, the quality of sleep, daytime sleepiness, clinical and echocardiographic parameters accompanied the improvement in the baroreflex sensitivity (BRS). In addition, among the analyzed breathing disorders during sleep, the presence of the central type of sleep apnea was associated with the greatest benefits of using CRT.

\section{Introduction}

Heart failure is a major and constantly increasing health problem. Currently the condition affects at least 26 million people worldwide [1]. Patients with chronic heart failure commonly suffer from breathing disorders during sleep [2-4]. It is estimated that sleep apnea occurs in $47-76 \%$ of patients with heart failure [5]. Heart failure is the principal risk factor of sleep breathing disorders, which may create a vicious circle of negative effects on the cardiovascular system [6]. The presence of breathing disorders during sleep in patients with heart failure is associated with increased risk of cardiovascular morbidity and mortality $[7,8]$.

\section{Corresponding author:}

Anna Przybyła MD, PhD, $1^{\text {st }}$ Department of Cardiology, Interventional Electrocardiology and Arterial Hypertension, Jagiellonian University Medical College, 17 Mikołaja Kopernika St, 31-501 Krakow, Poland, phone: +48 1242473 00, e-mail: przybylanka@op.pl

Received: 15.04.2019, accepted: 13.06.2019. 
The development of cardiac resynchronization therapy (CRT), which improves the exercise capacity, reduces the frequency of hospitalization associated with exacerbation of heart failure, reduces mortality, and improves the quality of life, has been a breakthrough in the treatment of heart failure [9-11]. The favorable effect of CRT on the prognosis and comfort of life may also be related to its impact on numerous disorders that accompany heart failure.

\section{Aim}

The aim of the present study was to assess the impact of CRT on sleep breathing disorders, and to determine the relationship between the changes observed in the autonomous nervous system and sleep disorders after the introduction of this therapy.

\section{Material and methods}

The study covered a group of 55 patients with chronic heart failure stable for at least the last 3 months, in New York Heart Association (NYHA) class III or IV despite optimal medical therapy, with a reduced left ventricular ejection fraction (LVEF) $\leq 35 \%$, QRS complex duration $\geq 120 \mathrm{~ms}$, and sinus rhythm. Patients who did not agree to participate in the study, had atrial fibrillation/flutter, had a sinus node disease, second or third degree atrioventricular block, implanted pacemaker, had numerous ventricular or supraventricular ectopic beats (more than $5 \%$ of all QRS complexes recorded), were hospitalized due to unstable angina, myocardial infarction or heart failure in the period of 3 months before implantation of the cardiac resynchronization system, underwent coronary artery bypass grafting or percutaneous coronary intervention within 3 months before implantation of the cardiac resynchronization system, underwent heart transplant surgery, had peripheral neuropathy, had contraindications for surgical procedures, who were treated for sleep disorders, and who participated in another research program at the time of the start of the study were excluded. In the case of coherence between RR interval and systolic blood pressure less than 0.5 , patients were excluded from the research, too. In addition, pregnant women and patients whose heart failure was secondary to reversible conditions were excluded from the study.

The study was conducted according to the principles of the Helsinki Declaration, and it was approved by the Institutional Ethical Committee. All enrolled patients were informed about the course of the study, and written informed consent for participation in this study was then obtained.

Before the cardiac resynchronization system was implanted and after 3 months of observation, each patient underwent an interview and physical examination, electrocardiography at rest, echocardiography, 6-minute walk test (6MWT) and polysomnography with the Pittsburgh
Sleep Quality Index (PSQI) questionnaire and the Epworth Sleepiness Scale (ESS) completed by each subject. Also baroreflex sensitivity (BRS) was evaluated. An X-ray scan of the chest was performed before and 3 days after implementation of CRT. The PSQI questionnaire evaluated the following 7 sleep quality parameters: time needed to fall asleep, duration and effectiveness of sleep, sleep disorders, the use of sleeping pills, subjective sleep quality, and difficulties with daytime functioning. The result of each sleep quality parameter ranged from 0 to 3 points. The maximum score was 21 . A result above 5 points indicated poor quality of sleep [12]. Additionally, the ESS was used, which allowed the assessment of the probability of falling asleep in eight different situations occurring in everyday life. A response to each question was given according to a 4-point scale (0-3), depending on the probability of falling asleep. The total score ranged between 0 and 24. A total score higher than 10 points indicated subjective excessive daytime sleepiness [13-15]. The sleep examination was performed in a hospital setting, in a single room, at night, using the Embla S 4000 polysomnographic system. The Somnologica software was used for the analysis of obtained data. The polysomnographic examination involved: single-channel electrocardiography, 3-channel electromyography, 2-channel electrooculography, 6-channel electroencephalography, and registration of the sound of snoring and air-flow through the nose/mouth. The patient's body position, movements of the chest and abdomen, as well as pulse oximetry were also monitored. Apnea was defined as an episode lasting for at least $10 \mathrm{~s}$ of a reduction in air-flow amplitude by $\geq 90 \%$ of the pre-event baseline. Apnea episodes were divided into: obstructive, central, and of mixed background. The classification was based on the recorded activity of respiratory muscles. Obstructive sleep apnea was diagnosed when an episode of a reduction in air-flow amplitude by $\geq 90 \%$ of pre-event baseline lasting for at least $10 \mathrm{~s}$ was accompanied by respiratory movements of the chest and abdomen. Central sleep apnea was diagnosed in the case of an episode of a reduction in air-flow amplitude by $\geq 90 \%$ of the pre-event baseline lasting for at least $10 \mathrm{~s}$ with no respiratory movements of the chest and abdomen. Mixed sleep apnea was defined as a combination of central sleep apnea at the initial phase and obstructive sleep apnea in the final phase. Hypopnea was defined as a reduction of the amplitude of air-flow by at least $30 \%$ of the pre-event baseline lasting for at least $10 \mathrm{~s}$, accompanied by oxygen desaturation by $\geq 3 \%$ from the pre-event baseline or arousal [16]. The following polysomnographic parameters were analyzed: apnea-hypopnea index $(\mathrm{AHI})$ indicating the number of apnea and hypopnea episodes per hour of sleep, apnea index (Al) corresponding to the number of apnea episodes per hour of sleep, obstructive apnea index (OAI) indicating the number of obstructive apnea episodes per hour of sleep, 
central apnea index (CAI) indicating the number of central apnea episodes per hour of sleep, mixed apnea index (MAI) indicating the number of mixed apnea episodes per hour of sleep, hypopnea index $(\mathrm{HI})$ indicating the number of hypopnea episodes per hour of sleep. The examination of BRS was carried out in a quiet room, after a 15-minute rest in a lying position, for stabilization of the cardiovascular system. All subjects refrained from eating, drinking coffee and smoking for $3 \mathrm{~h}$ before the start of the examination. The protocol involved registration of signals in a lying position with spontaneous respiration for $10 \mathrm{~min}$. Arterial blood pressure was monitored using the beat-tobeat method (Finometer Pro device - Finapres Medical Systems B.V.). An electrocardiogram was recorded, too. Baroreflex sensitivity was evaluated with non-invasive methods: spectral analysis and the sequence method (BRSseq). The $\alpha$ coefficient for the LF component (spectral power at low frequencies ranging between 0.04 and $0.14 \mathrm{~Hz}$ ) and for the HF component (spectral power at high frequencies ranging between 0.15 and $0.4 \mathrm{~Hz}$ ) was calculated as the square root of the quotient of the spectral power variability of the RR interval and systolic blood pressure. The transfer function modulus (TF) was calculated between RR interval oscillations and oscillations of systolic blood pressure for the LF and HF components. Assessment of baroreflex sensitivity using the sequential technique was based on the automatic capture of sequences of four or more consecutive heart evolutions, where the increase or decrease in systolic blood pressure was accompanied by a corresponding gradual increase or decrease of the length of the heart cycle. The slope of the regression curve plotted for the correlation between changes in systolic blood pressure and changes in the RR interval was assumed as the value of BRSseq [17].

\section{Statistical analysis}

The statistical analysis was performed using the Statistica 10 software and was presented as mean value \pm standard deviation (SD), median with interquartile range $(\mathrm{Q} 1-\mathrm{Q} 3)$ or percentages. Parameters were tested for normal distribution with the Shapiro-Wilk test. Data were analyzed using the Wilcoxon signed-rank test, the Mann-Whitney $U$ test and Spearman's rank correlation coefficient. A $p$-value of $<0.05$ was considered statistically significant.

\section{Results}

Fifty-five patients were enrolled in the study, including 45 males ( $81.8 \%$ of subjects) and 10 females (18.2\% of subjects). The clinical characteristic and pharmacotherapy of the study population are presented in Table I.

After a 3-month observation period, the QRS complexes narrowed (170 (160-200) vs. 140 (120-160) ms, $p<0.001)$ and also the echocardiography showed a significant improvement in LVEF (26\% (21-32\%) vs. $32 \%$
(27-37\%), $p<0.001)$. In addition, the severity of heart failure assessed according to the NYHA classification significantly decreased (3.11 \pm 0.31 vs. $2.2 \pm 0.58, p<$ $0.001)$ and the distance covered by the patients during the 6-minute walk test increased (300 (250-320) vs. 360 (300-400) $\mathrm{m}, p<0.001$ ).

In the study group, at baseline, $43.6 \%$ of patients had apnea that was predominantly central, $38.2 \%$ of patients had apnea that was predominantly obstructive and $18.2 \%$ of patients had apnea that was predominantly mixed. Polysomnography indicated a significant reduction in AHI after 3 months from CRT implementation. There was also a significant reduction in Al, CAl, and MAl. There were no significant changes in OAl or in HI. The evaluated poly-

Table I. Clinical characteristics and pharmacotherapy of the study group $(n=55)$

\begin{tabular}{|c|c|}
\hline Parameter & Results \\
\hline Male gender & 81.8 \\
\hline Age [years] & $67.04 \pm 9.13$ \\
\hline Body mass index $\left[\mathrm{kg} / \mathrm{m}^{2}\right]$ & $27.87 \pm 4.81$ \\
\hline Ischemic heart disease & 70.9 \\
\hline Dilated cardiomyopathy & 29.1 \\
\hline History of myocardial infarction & 61.8 \\
\hline History of percutaneous coronary intervention & 36.4 \\
\hline History of coronary artery bypass grafting & 16.4 \\
\hline Left bundle branch block & 67.3 \\
\hline Arterial hypertension & 69.1 \\
\hline Hypercholesterolemia & 78.2 \\
\hline Diabetes & 38.2 \\
\hline History of stroke & 7.3 \\
\hline Chronic kidney disease & 32.7 \\
\hline Anemia & 5.5 \\
\hline Hyperuricemia & 29.1 \\
\hline Currently smoking & 16.4 \\
\hline Smoking in the past & 25.5 \\
\hline ACEI/ARB & 92.7 \\
\hline$\beta$-Blocker & 100 \\
\hline Loop diuretics & 90.9 \\
\hline MRA & 67.3 \\
\hline Statin & 83.6 \\
\hline ASA & 74.5 \\
\hline
\end{tabular}

Data presented as mean value \pm standard deviation (SD) or percentage of patients (\%). ACEI - angiotensin-converting enzyme inhibitor, $A R B$ - angiotensin receptor blocker, ASA - acetylsalicylic acid, MRA - mineralocorticoid receptor antagonist. 
Table II. Polysomnographic parameters evaluated before and 3 months after implementation of cardiac resynchronization therapy

\begin{tabular}{lccc} 
Parameter & Baseline & Follow-up & P-value \\
\hline AHI [events/h] & $33.4(19.2-44.5)$ & $23.4(8.5-33.25)$ & $<0.001$ \\
\hline Al [events/h] & $23.22(11.1-31.31)$ & $12.77(2.89-21)$ & $<0.001$ \\
\hline OAl [events/h] & $3.89(2.04-10.97)$ & $3.43(1.22-9.41)$ & 0.067 \\
\hline CAl [events/h] & $6.01(1.52-11.92)$ & $0.45(0-4.31)$ & $<0.001$ \\
\hline MAl [events/h] & $4.4(1.24-9.66)$ & $2.99(0.27-9.4)$ & 0.009 \\
\hline HI [events/h] & $8.12(4.35-14.2)$ & $7.03(2.85-15.03)$ & 0.11
\end{tabular}

Data presented as median (Q1-Q3). AHI - apnea-hypopnea index, Al - apnea index, CAl - central apnea index, HI - hypopnea index, MAI - mixed apnea index, $O A I$-obstructive apnea index.

Table III. Sleep quality and daytime sleepiness before and 3 months after implantation of the cardiac resynchronization system

\begin{tabular}{lccc} 
Parameter & Baseline & Follow-up & $P$-value \\
\hline PSQI & $11(8-13)$ & $5(3-7)$ & $<0.001$ \\
\hline ESS & $11(7-13)$ & $7(4-10)$ & $<0.001$
\end{tabular}

Data presented as median (Q1-Q3). ESS - Epworth Sleepiness Scale, PSQI Pittsburgh Sleep Quality Index.

somnographic parameters are presented in Table II. An improvement defined as $50 \%$ or more reduction in the number of obstructive sleep apnea episodes was reported in $23.8 \%$ of patients with this type of sleep breathing disorders. There were no statistically significant differences in baseline LVEF ( $p=0.364)$, NYHA class $(p=0.283)$, 6MWT $(p=0.409)$, or body mass index $(\mathrm{BMI})(p=0.433)$ between groups of patients with obstructive sleep apnea responding and not responding to cardiac resynchronization therapy according to the accepted improvement criterion. In the examined group of patients, before the use of CRT, the subjects with $\mathrm{AHI}$ exceeding 30 events per hour of sleep prevailed. After the cardiac resynchronization system was implanted, the dominant group consisted of patients with $\mathrm{AHI}$ of 15-30 events per hour of sleep. Moreover, introduction of CRT resulted in significant improvement of the quality of sleep assessed with the PSQI questionnaire, and also in significant reduction of daytime sleepiness as measured with the ESS (Table III).

In the analysis of interrelations between the assessed parameters, statistically significant negative correlations between changes in $\mathrm{AHI}, \mathrm{Al}, \mathrm{CAl}$ and changes in BRS evaluated with all three methods in the supine position during spontaneous breathing were demonstrated. Also the relationships between changes in MAl and changes in BRSseq, $\alpha \mathrm{HF}$ and TF HF were statistically significant. Among the analyzed types of sleep breathing disorders, the strongest negative correlations were observed between changes in CAI and changes in BRS evaluated with all three methods. Significant negative correlations were observed between changes in intensity of daytime sleepiness and changes in BRS. In addition, significant negative correlations between changes in sleep quality and changes in the values of BRSseq, $\alpha \mathrm{HF}$, and TF HF were noted in the study group. However, no significant correlations were found between changes in sleep quality and changes in $\alpha$ LF and TF LF values. Statistically significant positive correlations were observed between changes in LVEF, 6MWT and changes in BRSseq, $\alpha$ HF, TF HF. There were significant negative correlations between changes in NYHA class and changes in BRSseq, $\alpha \mathrm{HF}$, TF HF. Non-significant correlations were noted between changes in LVEF, NYHA class, 6MWT and changes in $\alpha$ LF, TF LF. The results mentioned above are presented in Table IV.

The mutual associations between changes in polysomnographic parameters and changes in the quality of sleep, daytime sleepiness, LVEF, NYHA class, 6MWT occurring after 3 months of CRT are presented in Table V. It was observed that changes in $\mathrm{AHI}, \mathrm{Al}$ and $\mathrm{CAl}$ were significantly correlated with changes in the quality of sleep, daytime sleepiness and changes in LVEF, NYHA class, and $6 \mathrm{MWT}$. Moreover, there was a statistically significant positive correlation between changes in MAl and changes in the subjective assessment of sleep quality. However, there were no significant correlations between changes in OAI, MAI, HI and changes in LVEF, NYHA class, 6MWT, as well as between changes in $\mathrm{OAI}, \mathrm{HI}$ and changes in the quality of sleep, and also between changes in OAI, MAI, $\mathrm{HI}$ and changes in daytime sleepiness.

There were statistically significant negative correlations between changes in the quality of sleep and changes in LVEF and 6MWT, as well as a statistically significant positive correlation between changes in the quality of sleep and changes in NYHA class. Also, changes in the severity of daytime sleepiness correlated negatively, significantly with changes in LVEF. The results are shown in Table VI.

\section{Discussion}

In the present study, the values of $\mathrm{AHI}, \mathrm{Al}, \mathrm{CAl}$, and MAI were significantly reduced after 3 months of CRT. 
Table IV. Correlations between changes in baroreflex sensitivity measured by the sequence method, the $\alpha$ coefficient, the transfer function modulus and changes in the polysomnographic parameters, daytime sleepiness, sleep quality, left ventricular ejection fraction, NYHA class, 6-minute walk test

\begin{tabular}{|c|c|c|c|c|c|c|c|c|c|c|}
\hline \multirow[t]{2}{*}{ Parameter } & \multicolumn{2}{|c|}{$\Delta$ BRSseq $[\mathrm{ms} / \mathrm{mm} \mathrm{Hg}]$} & \multicolumn{2}{|c|}{$\Delta \alpha \mathrm{LF}[\mathrm{ms} / \mathrm{mm} \mathrm{Hg}]$} & \multicolumn{2}{|c|}{$\Delta \alpha \mathrm{HF}[\mathrm{ms} / \mathrm{mm} \mathrm{Hg}]$} & \multicolumn{2}{|c|}{$\Delta \mathrm{TF} \mathrm{LF}[\mathrm{ms} / \mathrm{mm} \mathrm{Hg}]$} & \multicolumn{2}{|c|}{$\Delta \mathrm{TF} \mathrm{HF}[\mathrm{ms} / \mathrm{mm} \mathrm{Hg}]$} \\
\hline & $r$ & $P$-value & $r$ & $P$-value & $r$ & $P$-value & $r$ & $P$-value & $r$ & $P$-value \\
\hline$\Delta \mathrm{AHI}[$ events/h] & -0.442 & $<0.001$ & -0.304 & 0.024 & -0.518 & $<0.001$ & -0.299 & 0.027 & -0.524 & $<0.001$ \\
\hline$\Delta \mathrm{Al}[$ events $/ \mathrm{h}]$ & -0.485 & $<0.001$ & -0.331 & 0.014 & -0.523 & $<0.001$ & -0.392 & 0.003 & -0.521 & $<0.001$ \\
\hline$\Delta \mathrm{OAl}[$ events/h] & -0.257 & 0.058 & -0.091 & 0.509 & -0.164 & 0.233 & -0.154 & 0.261 & -0.197 & 0.149 \\
\hline$\Delta \mathrm{CAl}[$ events/h] & -0.385 & 0.004 & -0.277 & 0.04 & -0.469 & $<0.001$ & -0.333 & 0.013 & -0.482 & $<0.001$ \\
\hline$\Delta \mathrm{MAl}[$ events/h] & -0.374 & 0.005 & -0.193 & 0.158 & -0.296 & 0.028 & -0.236 & 0.083 & -0.328 & 0.014 \\
\hline$\Delta \mathrm{HI}[$ events/h] & -0.084 & 0.541 & -0.061 & 0.659 & -0.101 & 0.465 & -0.021 & 0.881 & -0.168 & 0.221 \\
\hline$\Delta \mathrm{ESS}$ & -0.368 & 0.006 & -0.321 & 0.017 & -0.435 & $<0.001$ & -0.315 & 0.019 & -0.445 & $<0.001$ \\
\hline$\triangle \mathrm{PSQ}$ & -0.338 & 0.012 & -0.213 & 0.118 & -0.342 & 0.011 & -0.18 & 0.189 & -0.349 & 0.009 \\
\hline$\Delta$ LVEF $[\%]$ & 0.505 & $<0.001$ & 0.246 & 0.07 & 0.413 & 0.002 & 0.176 & 0.198 & 0.368 & 0.006 \\
\hline$\Delta$ NYHA class & -0.304 & 0.024 & -0.152 & 0.267 & -0.44 & $<0.001$ & -0.212 & 0.12 & -0.432 & $<0.001$ \\
\hline$\Delta$ 6MWT $[\mathrm{m}]$ & 0.288 & 0.033 & 0.205 & 0.134 & 0.424 & 0.001 & 0.197 & 0.15 & 0.479 & $<0.001$ \\
\hline
\end{tabular}

AHI-apnea-hypopnea index, Al-apnea index, $\alpha H F-\alpha$ coefficient in the high frequency band, $\alpha L F-\alpha$ coefficient in the low frequency band, BRSseq-baroreflex sensitivity measured by the sequence method, CAI - central apnea index, ESS - Epworth Sleepiness Scale, HI - hypopnea index, LVEF - left ventricular ejection frac tion, MAI - mixed apnea index, NYHA - New York Heart Association, OAI - obstructive apnea index, PSQI - Pittsburgh Sleep Quality Index, 6MWT - 6-minute walk test, TF HF-transfer function modulus in the high frequency band, TF LF-transfer function modulus in the low frequency band.

Table V. Correlations between changes in the objective sleep parameters and changes in the subjective sleep parameters, left ventricular ejection fraction, NYHA class, 6-minute walk test

\begin{tabular}{|c|c|c|c|c|c|c|c|c|c|c|}
\hline \multirow[t]{2}{*}{ Parameter } & \multicolumn{2}{|c|}{$\Delta \mathrm{ESS}$} & \multicolumn{2}{|c|}{$\triangle$ PSQI } & \multicolumn{2}{|c|}{$\Delta$ LVEF (\%) } & \multicolumn{2}{|c|}{$\Delta$ NYHA class } & \multicolumn{2}{|c|}{$\Delta$ 6MWT $[\mathrm{m}]$} \\
\hline & $r$ & $P$-value & $r$ & $P$-value & $r$ & $P$-value & $r$ & $P$-value & $r$ & $P$-value \\
\hline$\Delta \mathrm{AHI}[$ events/h] & 0.443 & $<0.001$ & 0.528 & $<0.001$ & -0.517 & $<0.001$ & 0.38 & 0.004 & -0.513 & $<0.001$ \\
\hline$\Delta \mathrm{Al}[$ events/h] & 0.413 & 0.002 & 0.533 & $<0.001$ & -0.553 & $<0.001$ & 0.378 & 0.004 & -0.508 & $<0.001$ \\
\hline$\Delta \mathrm{OAI}[$ events/h] & 0.172 & 0.208 & 0.235 & 0.084 & -0.139 & 0.31 & 0.195 & 0.154 & -0.134 & 0.329 \\
\hline$\Delta \mathrm{CAl}[$ events/h] & 0.437 & $<0.001$ & 0.373 & 0.005 & -0.524 & $<0.001$ & 0.372 & 0.005 & -0.57 & $<0.001$ \\
\hline$\Delta \mathrm{MAl}[$ events/h] & 0.257 & 0.058 & 0.276 & 0.042 & -0.213 & 0.119 & 0.202 & 0.14 & -0.101 & 0.465 \\
\hline$\Delta \mathrm{HI}[$ events/h] & 0.198 & 0.148 & 0.143 & 0.297 & -0.128 & 0.353 & 0.189 & 0.166 & -0.22 & 0.106 \\
\hline
\end{tabular}

AHI - apnea-hypopnea index, Al - apnea index, CAI - central apnea index, ESS - Epworth Sleepiness Scale, HI - hypopnea index, LVEF - left ventricular ejection fraction, MAI - mixed apnea index, NYHA - New York Heart Association, OAI - obstructive apnea index, PSQI - Pittsburgh Sleep Quality Index, 6MWT - 6-minute walk test.

Table VI. Correlations between changes in clinical and echocardiographic parameters and changes in subjective sleep parameters

\begin{tabular}{lcccccc} 
& \multicolumn{2}{c}{$\Delta$ LVEF (\%) } & \multicolumn{2}{c}{$\Delta$ NYHA class } & \multicolumn{2}{c}{$\Delta$ 6MWT [m] } \\
\cline { 2 - 7 } & $r$ & $P$-value & $r$ & $P$-value & $r$ & $P$-value \\
\hline$\Delta$ PSQI & -0.512 & $<0.001$ & 0.305 & 0.023 & -0.53 & $<0.001$ \\
\hline$\Delta$ ESS & -0.342 & 0.011 & 0.259 & 0.056 & -0.209 & 0.126
\end{tabular}

ESS - Epworth Sleepiness Scale, LVEF - left ventricular ejection fraction, NYHA - New York Heart Association, PSQI - Pittsburgh Sleep Quality Index, 6MWT - 6-minute walk test. 
Moreover, no significant changes in OAl and $\mathrm{HI}$ were noted. In the literature there are controversial data about the influence of CRT on sleep breathing disorders. Lüthje et al. demonstrated that CRT significantly reduces the central apnea-hypopnea index remaining without a significant impact on the obstructive apnea-hypopnea index [18]. By contrast, Stanchina et al. assessing the effect of CRT on obstructive respiratory events observed a decrease in $\mathrm{AHI}$ [19]. In addition, in the present study a statistically significant reduction in the number of episodes of central sleep apnea accompanied the improvement of LVEF. Significant negative correlations between changes in LVEF and changes in CAI, as well as between changes in LVEF and changes in $\mathrm{AHI}$ and $\mathrm{Al}$, were noted. However, there were no statistically significant relationships between changes in LVEF and changes in OAI, MAI, or HI. This suggests the existence of a relationship between the number of central sleep apnea episodes and the severity of heart failure.

The dysfunction of the autonomous nervous system may be manifested by a decrease in baroreflex sensitivity [20]. In the present study, negative correlations between changes in baroreflex sensitivity and changes in the polysomnographic parameters were demonstrated. Statistically significant negative correlations were found between changes in $\mathrm{AHI}, \mathrm{Al}$ and changes in baroreflex sensitivity evaluated using the sequence method, the $\alpha$ coefficient and the transfer function modulus. Among the analyzed types of sleep breathing disorders, the strongest correlations were demonstrated between changes in BRS and changes in CAI. The present results suggest that the improvement of sleep breathing disorders occurring 3 months after the implementation of CRT is parallel to the improvement of baroreflex sensitivity. Assessment of baroreflex sensitivity may therefore play an important role in predicting the severity of sleep breathing disorders. The significant reduction in the central apnea index with the strongest, among the analyzed types of sleep breathing disorders, statistically significant negative correlations between the changes in this index and changes in baroreflex sensitivity may indicate that the patients with heart failure and the central type of sleep apnea will benefit most from implantation of the cardiac resynchronization system.

An important problem in patients with heart failure is the poor quality of sleep, which is a barrier to self-care and compliance with treatment in this group of patients [21]. In the present study, the improvement of the polysomnographic parameters was accompanied by an improvement in the subjective quality of sleep assessed using the PSQI questionnaire. Statistically significant correlations between changes in sleep quality and changes in $\mathrm{AHI}, \mathrm{Al}$, and CAl were noted. A statistically significant, though weaker, correlation was observed between changes in the quality of sleep and changes in MAI. In addition to assessing the quality of sleep, the subjective assessment of daytime sleepiness is also an important element in the diagnosis of sleep disorders. Daytime sleepiness measured with the ESS was significantly decreased following CRT. An association between daytime sleepiness and baroreflex sensitivity was demonstrated in the present study. It was found that the reduction of daytime sleepiness after the use of CRT was simultaneously associated with increased baroreflex sensitivity. Statistically significant negative correlations were observed between changes in daytime sleepiness and changes in baroreflex sensitivity measured using the sequence method, the $\alpha$ coefficient and the transfer function modulus. Occurrence of excessive daytime sleepiness may indicate changes in autonomous regulation of the heart work in patients with sleep breathing disorders. The reduction of $\mathrm{AHI}$ and daytime sleepiness coexisting with the increase in BRS observed after the use of CRT indicates an increase in the activity of the parasympathetic part of the autonomous nervous system. Moreover, the obtained results suggest that an assessment of baroreflex sensitivity and the apnea-hypopnea index may be useful in predicting the occurrence of daytime sleepiness and its severity. It is worth noting that after the implementation of cardiac resynchronization therapy, the improvement of clinical and echocardiographic parameters was accompanied by an improvement in subjective sleep assessment, which intensified the benefits of this therapy.

The obtained results indicate that the use of cardiac resynchronization therapy may bring the greatest benefits in patients with heart failure and the coexisting central type of sleep apnea. Already after a 3-month observation period, the implementation of cardiac resynchronization therapy resulted in a significant reduction in the number of central sleep apnea episodes with simultaneous improvement in both clinical and echocardiographic parameters, subjective sleep parameters, as well as in the autonomous nervous system function. This suggests that the coexistence of central sleep apnea in patients with heart failure is a predictor of response to cardiac resynchronization therapy. Therefore, screening for sleep breathing disorders prior to implantation of a cardiac resynchronization system may help identify patients with heart failure who will respond to cardiac resynchronization therapy and will receive the greatest multidirectional benefits of this therapy. In addition, baroreflex sensitivity is a reflection of the clinical and echocardiographic response to CRT, as well as the severity of breathing disorders during sleep, sleep quality and daytime sleepiness, so it can be used to assess the effectiveness of cardiac resynchronization therapy.

\section{Conclusions}

The present study showed that the use of CRT reduced the number of apnea-hypopnea episodes, and 
in particular the number of central and mixed type sleep apnea episodes. Moreover, a beneficial effect of CRT not only on objective but also subjective sleep features, such as sleep quality and daytime sleepiness, was observed already after 3 months of this therapy. The improvement in sleep breathing disorders, the quality of sleep, daytime sleepiness, clinical and echocardiographic parameters occurring after the implementation of CRT was associated with the improvement of the autonomous nervous system function. Due to a significant reduction of CAl after the use of CRT, and the occurrence of the strongest among the analyzed types of sleep breathing disorders, statistically significant negative correlations between changes in CAl and changes in BRS, and also because of the strongest relationship between improvement in CAI and improvement in sleep quality, daytime sleepiness, clinical and echocardiographic parameters, it may be assumed that patients with heart failure and the central type of sleep apnea will benefit most from CRT.

\section{Acknowledgments}

This study was supported by the Ministry of Science and Higher Education (grant number K/ZDS/000557).

\section{Conflict of interest}

The authors declare no conflict of interest.

\section{References}

1. Savarese G, Lund LH. Global Public Health Burden of Heart Failure. Card Fail Rev 2017; 3: 7-11.

2. Matsumoto H, Kasai T, Suda S, et al. Randomized controlled trial of an oral appliance (SomnoDent) for sleep-disordered breathing and cardiac function in patients with heart failure. Clin Cardiol 2018; 41: 1009-12.

3. Rosen D, Roux FJ, Shah N. Sleep and breathing in congestive heart failure. Clin Chest Med 2014; 35: 521-34.

4. Parisi V, Paolillo S, Rengo G, et al. Sleep-disordered breathing and epicardial adipose tissue in patients with heart failure. Nutr Metab Cardiovasc Dis 2018; 28: 126-32.

5. Jaffe LM, Kjekshus J, Gottlieb SS. Importance and management of chronic sleep apnoea in cardiology. Eur Heart J 2013; 34: 809-15.

6. Wexler L, Javaheri S. Sleep apnea is linked to heart failure, but does treatment improve outcome? Cleve Clin J Med 2005; 72 929-36.

7. Vazir A, Sundaram V. Management of sleep apnea in heart failure. Heart Fail Clin 2018; 14: 635-42.

8. Farrell PC, Richards G. Recognition and treatment of sleep-disordered breathing: an important component of chronic disease management. J Transl Med 2017; 15: 114.

9. Przybyła A, Czarnecka D, Kusiak A, et al. The influence of cardiac resynchronization therapy on selected inflammatory markers and aldosterone levels in patients with chronic heart failure. Przegl Lek 2011; 68: 359-61.

10. Wiliński J, Czarnecka D, Wojciechowska W, et al. Clinical and classic echocardiographic features of patients with, and with- out, left ventricle reverse remodeling following the introduction of cardiac resynchronization therapy. Cardiol J 2011; 18: 157-64.

11. Almeida-Morais L, Abreu A, Oliveira M, et al. Impact of cardiac resynchronization therapy on inflammatory biomarkers and cardiac remodeling: the paradox of functional and echocardiographic response. Rev Port Cardiol 2018; 37: 105-13.

12. Buysse DJ, Reynolds CF $3^{\text {rd }}$, Monk TH, et al. The Pittsburgh Sleep Quality Index: a new instrument for psychiatric practice and research. Psychiatry Res 1989; 28: 193-213.

13. Mehra R, Wang L, Andrews N, et al. Dissociation of objective and subjective daytime sleepiness and biomarkers of systemic inflammation in sleep-disordered breathing and systolic heart failure. J Clin Sleep Med 2017; 13: 1411-22.

14. Johns MW. A new method for measuring daytime sleepiness: the Epworth sleepiness scale. Sleep 1991; 14: 540-5.

15. Johns MW. Daytime sleepiness, snoring, and obstructive sleep apnea. The Epworth Sleepiness Scale. Chest 1993; 103: 30-6.

16. Berry RB, Budhiraja R, Gottlieb DJ, et al. Rules for scoring respiratory events in sleep: update of the 2007 AASM Manual for the Scoring of Sleep and Associated Events. Deliberations of the Sleep Apnea Definitions Task Force of the American Academy of Sleep Medicine. J Clin Sleep Med 2012; 8: 597-619.

17. Parati G, Di Rienzo M, Mancia G. How to measure baroreflex sensitivity: from the cardiovascular laboratory to daily life. J Hypertens 2000; 18: 7-19.

18. Lüthje L, Renner B, Kessels R, et al. Cardiac resynchronization therapy and atrial overdrive pacing for the treatment of central sleep apnoea. Eur J Heart Fail 2009; 11: 273-80.

19. Stanchina ML, Ellison K, Malhotra A, et al. The impact of cardiac resynchronization therapy on obstructive sleep apnea in heart failure patients: a pilot study. Chest 2007; 132: 433-9.

20. Willis FB, Isley AL, Geda YE, et al. Resolution of syncope with treatment of sleep apnea. J Am Board Fam Med 2008; 21: 466-8.

21. Parati G, Lombardi C, Castagna F, et al. Heart failure and sleep disorders. Nat Rev Cardiol 2016; 13: 389-403. 